\title{
Valor de uso de un activo o unidad generadora de efectivo bajo incertidumbre: el flujo de efectivo esperado mediante metodología borrosa*
}

doi:10.11144/Javeriana.cc17-44.vuau

Fabián Alberto Castiblanco-Ruiz

Estudiante Estudiante de doctorado en ingeniería

matemática, estadística e investigación de operaciones,

Universidad Complutense de Madrid, España. Magíster en

matemáticas de la Universidad de Sevilla, España. Licen-

ciado en matemáticas, Universidad Pedagógica Nacional,

Colombia. Docente investigador, Facultad de Contaduría

Pública, Universidad La Gran Colombia, sede Bogotá.

Líder del grupo de investigación Globalización, Gestión y

Organizaciones. Director del semillero de investigación

ICOLDI, Investigación Contable y Lógica Difusa.

Correo electrónico: fabianalberto.castiblanco@ugc.edu.co

\footnotetext{
* Artículo de reflexión producto de la investigación Avances frente al concepto de valor en contabilidad y el proceso de valoración contable a partir de la lógica difusa, financiado y desarrollado por la Facultad de Contaduría Pública de la Universidad La Gran Colombia, UGC, sede Bogotá durante el período 2014-2, 2015-1 y bajo el código de registro FCP/2015/001.
} 
Resumen En el estudio en prospectiva de las organizaciones se hace necesaria la consideración y tratamiento de la incertidumbre y subjetividad propia de los fenómenos económicos y financieros. En el presente artículo se busca establecer los procedimientos y aportes del uso de una metodología basada en la teoría de los subconjuntos borrosos que permite, en particular, el tratamiento de un tipo de incertidumbre específica en la estimación del valor de uso de un activo o unidad generado de efectivo.

Para tal fin, se acude al uso de tres elementos fundamentales: el establecimiento de una tipología sobre la existencia de incertidumbre, las herramientas propias de los subconjuntos borrosos y los lineamientos dados por la Norma Internacional de Contabilidad 36. Se establecen las posibles relaciones entre los conceptos abordados y se destacan los aportes y ventajas de esta relación.

Palabras claves Valor de uso; flujo de efectivo esperado; incertidumbre; subconjuntos borrosos

Códigos JEL M40, M41, M48, C6

\section{Value in Use of an Asset or Cash- Generating Unit Under Uncertainty: Expected Cash Flow Through a Fuzzy Methodology}

\footnotetext{
Abstract In the prospective study of organizations it becomes necessary to consider and handle the uncertainty and subjectivity characteristic of economic and financial phenomena. This paper aims to establish the procedures and contributions of the use of a methodology based on the theory of the fuzzy subsets which would allow, specifically, treating a specific type of uncertainty in the estimation of the value of use of an asset or cash-generating unit. For this purpose, three key elements are used: the establishment of a typology on the existence of uncertainty; the tools particular to fuzzy subsets; and the guidelines given by the International Accounting Standard 36. The possible relationships between the concepts discussed are estab-
}

lished and we highlight the contributions and advantages of this relationship.

Keywords value in use; expected cash flow; uncertainty; fuzzy subsets

\section{Valor em uso de um ativo ou unidade geradora de caixa sob incerteza: o fluxo de caixa esperado utilizando metodologia difusa}

Resumo No estudo em prospectiva das organizações faz-se necessária a consideração e tratamento da incerteza e subjetividade própria dos fenómenos económicos e financeiros. No presente artigo visa-se estabelecer os procedimentos e contribuições do uso de metodologia baseada na teoria dos subconjuntos difusos que permitem, em particular, o tratamento de um tipo de incerteza específica na estimativa do valor em uso de um ativo ou unidade gerada de caixa. Para isso, acode-se ao uso de três elementos fundamentais: o estabelecimento de uma tipologia sobre a existência de incerteza, as ferramentas próprias dos subconjuntos difusos e as diretrizes pela Norma Internacional de Contabilidade 36. Estabelecem-se as possíveis relações entre os conceitos abordados e salientam-se as contribuições e vantagens desta relação.

Palavras-chave Valor de uso; fluxo de caixa esperado; incerteza; subconjuntos difusos

\section{Introducción}

Las ciencias sociales, en particular en el ámbito económico, administrativo y contable, reflexionan acerca de los elementos constitutivos de las organizaciones, sus interrelaciones, sus devenires y estructuras dinámicas, como fenómenos de naturaleza social que están mediados por los sujetos y sus relaciones, por su micro y 
macroentorno. En este sentido, se estudian las decisiones que enfrentan y sus consecuencias, se analiza el contexto en el cual está inmerso, las relaciones de poder y de jerarquización existentes, sus relaciones con el entorno, las intencionalidades de sus actores y el entramado complejo que de ellas emerge de manera continua.

Desde la anterior perspectiva, un elemento determinante en el análisis de los fenómenos sociales propios de las organizaciones es el estudio, comprensión y diagnóstico de la evolución futura de las variables que lo componen: ventas, costos, inversiones, pasivos, estructura administrativa, impacto, crecimiento, flujos de efectivo, por nombrar algunas. En particular, la proyección financiera como herramienta de diagnóstico sobre la actuación de la organización ante los cambios propios y del entorno, es un componente fundamental en el proceso de toma de decisiones.

Por lo tanto, se convierte tanto en desafío como en necesidad, establecer un imaginario sobre el comportamiento futuro y sobre las alternativas que la organización pueda presentar en prospectiva y sus variables.

Ante tal desafío, los desarrollos académicos y necesariamente interdisciplinares plantean y estructuran herramientas que permiten establecer modelos, simulaciones o predicciones de acontecimientos futuros, de acuerdo con las características del entorno y el comportamiento de las variables que intervienen. Estas predicciones pueden plantearse desde una doctrina determinística, en la cual el futuro se comporta de manera lineal con relación al pasado o está preestablecido a partir de causas identificadas. Por otro lado, puede concebirse la evolución o comportamiento futuro de ciertas variables desde una perspectiva compleja, en la cual el azar y los sucesos imprevisibles son el rasgo principal.

Cualquiera que sea la postura, la incertidumbre inherente a los acontecimientos futuros se convierte en el elemento principal a ser tratado. En algunos casos, se reduce e incluso se desecha, debido a la percepción lineal del comportamiento de las variables; en otros casos, se modela mediante probabilidades de ocurrencia o comportamiento de ellas; y en algunos otros, la información limitada o el desconocimiento profundo de las variables hacen que la incertidumbre no pueda ser tratada adecuadamente.

En particular, analizar el valor del dinero en el tiempo pone en juego variables localizadas en el futuro y cuyos valores están cargados de incertidumbre. De manera específica, el concepto de valor presente de una cantidad monetaria, como variable del dinero en el tiempo, requiere el trabajo con cifras estimadas cuya certeza o probabilidad de ocurrencia no están siempre determinadas o no es posible establecer.

En este sentido, el presente artículo propone abordar y tratar una clase específica de incertidumbre que emerge cuando se calcula el valor de uso de un activo o unidad generadora de efectivo (UGE) ${ }^{1}$ bajo ciertas condiciones. En particular, se estudian las estimaciones planteadas en el cálculo del valor presente del flujo de caja proyectado o flujo de caja esperado. Por lo tanto, este documento es el resultado parcial de

1 Valor de uso es el valor actual de los flujos futuros de efectivo estimados que se espera obtener de un activo o unidad generadora de efectivo. 
un ejercicio investigativo en el cual se aplica la teoría de los subconjuntos borrosos en la determinación del valor de uso y sus implicaciones en la estimación de la pérdida por deterioro del valor del activo o UGE. Este ejercicio investigativo, en últimas, permitirá reconocer las ventajas de aplicar la teoría y describir su uso.

La metodología empleada para tal objetivo corresponde a los elementos propios de una investigación correlacional y experimental, en la cual se establecen los resultados de la aplicación de los subconjuntos borrosos en la determinación del valor de uso de un activo o UGE.

Esta metodología busca establecer un punto de articulación entre los conceptos de subconjunto borroso y valor de uso, a partir de las categorías de incertidumbre y subjetividad; tales categorías permiten el tratamiento de estimaciones sobre valores monetarios futuros dados desde la aproximación o posibilidad de ocurrencia. Por lo tanto, mediante los procesos de borrosificación y desborrosificación (propios de la lógica borrosa, como marco general de la teoría de los subconjuntos borrosos) se simulan y tratan las variables desde las categorías iniciales. Finalmente, se evalúan las ventajas y aportes hallados mediante la relación planteada entre los dos conceptos.

Según lo anterior, en la primera parte del documento se analizan, a la luz de las categorías de incertidumbre y subjetividad, los presupuestos financieros como base del concepto de valor de uso. Posteriormente, en los aspectos metodológicos se describen los conceptos necesarios de la teoría de los subconjuntos borrosos y los procesos de borrosificación y desborrosificación. En la sección de resultados se presenta un ejemplo de aplicación que permite identificar los aportes y ventajas de esta correlación. Al finalizar, se plantean las conclusiones del ejercicio.

\section{Las categorías de incertidumbre y subjetividad en el proceso de estimación del valor de uso}

En el ámbito de la gestión organizacional, se hace predominante el establecimiento de un proceso de planificación que permita la consecución de los objetivos trazados por la organización. En esta planificación, se contempla la elaboración de los presupuestos financieros definidos como la representación de las “[...] estimaciones de todas las transacciones de inversiones, ingresos y gastos para un período contable subsiguiente, incluyendo estados proforma o proyectados" (Cárdenas y Napoles, 2002, p. 2). En particular, esta planificación permite por medio de los presupuestos o previsiones financieras estimar el valor de uso de un activo o UGE.

La preparación de los presupuestos financieros se inicia generalmente con la asignación de valores (en términos monetarios) que expresen los objetivos propuestos por la organización. Es decir, mediante estimaciones se plantea una relación entre una escala de medida y un hecho localizado en el futuro. Por lo tanto, esta situación corresponde con un proceso de valuación, en el cual intervienen cifras que buscan modelar acontecimientos en prospectiva y dependen de intereses particulares.

En términos generales, ante cualquier procedimiento de valuación, Richard Mattessich 
(2002) establece que el proceso de asignación está permeado por la influencia de la subjetividad y por los continuos cambios de la escala seleccionada. Es decir, se pone de manifiesto la presencia de juicios de valor provenientes de la racionalidad emocional, incluso si están en relación directa con un agregado social como el mercado. Por otra parte, se reconoce la variabilidad propia de las cifras en entornos económicos, máxime si se plantean a futuro.

Así pues, los presupuestos financieros están permeados por dos elementos inherentes a su naturaleza: la incertidumbre y la subjetividad. En particular, la incertidumbre presenta algunos matices que se hace necesario distinguir.

En términos generales, a partir del desarrollo de la teoría de la incertidumbre (Gil-Aluja, 2000), en el ámbito económico y empresarial se distingue entre dos tipos de incertidumbre: la incertidumbre epistémica y la incertidumbre óntica.

En esta aproximación, la incertidumbre epistémica se concibe como la ausencia de conocimiento claro, seguro y evidente, bien sea objetiva o subjetivamente, es decir, por el estado psicológico del sujeto que se cree o no en posesión de tal conocimiento; mientras que la incertidumbre óntica se refiere a lo incierto en los hechos o entes (Pérez, 1999; Ramírez-Sarrió, 1988).

La teoría de la incertidumbre busca el tratamiento particular del enfoque epistémico. En este marco de referencia, se hace alusión a la falta de seguridad sobre la verdad del conocimiento y a la imposibilidad de establecer una medida por medio de la probabilidad. Se establece, por lo tanto, una incertidumbre caracterizada por la ausencia de conocimiento perfecto, por la presencia de inexactitud de la representación pretendida y/o alcanzada de un concepto o ente, la vaguedad en la verdad de un enunciado o la aproximación de tal enunciado (Castiblanco-Ruiz, 2016).

En la misma línea, Camilo A. Franco de los Ríos (2012) y Álvaro Torres y Carolina Tranchita (2004) se refieren a estos conceptos mediante la identificación de un sistema de inexactitud natural cuyas formas pueden estar dadas por la vaguedad, es decir, por conceptos que no tienen límites o fronteras bien definidas, pues su falsedad o verdad no se puede establecer con exactitud. De igual forma, pueden estar dadas por la ambigüedad, cuando hay diferentes significados para un mismo concepto o, pueden estar dadas por la ambivalencia, referida a la existencia de nociones antagónicas o conflictivas.

Desde la anterior perspectiva, la norma internacional de contabilidad 36 (NIC 36) (IFRS Foundation, 2014) en su apéndice A, aborda y plantea las metodologías para estimar los flujos de efectivo futuros. Se destaca el enfoque de flujo de efectivo esperado como una técnica que permite considerar todas las expectativas de los posibles flujos de efectivo. En particular, se establece esta técnica en situaciones específicas cargadas de incertidumbre; "el enfoque del flujo de efectivo esperado permite también usar las técnicas del valor actual cuando la distribución temporal de los flujos de efectivo está sometida a incertidumbre" (párrafo A8).

Frente a lo anterior, la NIC 36 diferencia dos tipos de situaciones; la primera abarca los contextos donde los posibles flujos de efectivo esperado se determinan mediante una distribución de probabilidad. La segunda contempla los escenarios en los cuales la información sobre 
estas distribuciones es limitada o imposible de establecer, es decir, situaciones en el marco de incertidumbre epistémica.

A su vez, la NIC 36 propone un tratamiento particular de esta información, que se describe a continuación:

Un contable podría encontrarse ante las siguientes situaciones:

a) El importe estimado se sitúa en algún lugar entre 50 u.m. y 250 u.m., sin que ningún importe dentro del rango sea más probable que cualquier otro. A partir de esa información limitada, el flujo de efectivo esperado es de 150 u.m. [(50 + 250)/2].

b) El importe estimado se sitúa en algún lugar entre 50 u.m. y 250 u.m.; el importe más probable es 100 u.m. Sin embargo, las probabilidades asociadas a cada importe son desconocidas. A partir de esa información limitada, el flujo de efectivo esperado es 133,33 u.m. [(50+100+250)/3].

c) El importe estimado será 50 u.m. (con el 10\% de probabilidad), 250 u.m. (con el $30 \%$ de probabilidad), o 100 u.m. (con el $60 \%$ de probabilidad). A partir de esa información limitada, el flujo de efectivo esperado es 140 u.m. [(50 x 0,10) + (250 x $0,30)+(100 \times 0,60)]$.

En cada caso, el flujo de efectivo estimado esperado proporcionará, con toda probabilidad, una mejor estimación del valor en uso que los importes mínimo, más probable o máximo tomados de forma aislada (párrafo A11).

En la misma norma, se plantean las dificultades que este tipo de mediciones puede generar en el establecimiento de estimaciones fiables $y$, aunque consideren la incertidumbre y la subjetividad, pueden arrojar valores alejados de la realidad y no representativos.

En particular, con base en la perspectiva teórica empleada sobre incertidumbre, se identifica que situaciones como las descritas en los literales (a) y (b) corresponden a incertidumbre epistémica, en las cuales hay un conocimiento escaso o nulo sobre las probabilidades de ocurrencia de ciertos valores o mejor aún, el conocimiento sobre los valores presenta las imperfecciones propias del hecho observado.

Por lo tanto, se busca presentar una alternativa que permita tratar y abordar la incertidumbre epistémica emanada de la información disponible en el establecimiento del flujo de efectivo esperado mediante las herramientas borrosas. En particular, la teoría de los subconjuntos borrosos presenta sólidos sustentos matemáticos, pues permite realizar un tratamiento de la información cargada de incertidumbre y en la cual no se cuenta con la información suficiente para establecer funciones de probabilidad sobre el acaecimiento de los sucesos. A partir de este tratamiento, se presentan las ventajas obtenidas de la relación establecida.

Con un caso particular se expone la metodología empleada y se ilustra el tipo de información sobre el cual se aplica la teoría. La ejemplificación se hace necesaria en cuanto a proceso de experimentación se refiere; la teoría de los subconjuntos borrosos se aplica bajo ciertas condiciones iniciales ante la identificación de una incertidumbre particular.

Según lo anterior, la presencia de incertidumbre en la determinación del valor de uso de un activo o UGE se refiere, en muchas ocasiones 
a una incertidumbre epistémica, pues el flujo de caja proyectado se establece como variable del valor de uso y se parte de estimaciones cargadas de subjetividad como proceso de valuación.

\section{Aspectos metodológicos}

El uso y aplicación de la teoría de los subconjuntos borrosos en el estudio de fenómenos o conceptos propios de las ciencias económicas administrativas o contables, requiere la identificación adecuada de los conceptos y variables a ser tratadas; en particular, se requiere evaluar la pertinencia y validez de los aspectos a relacionar y los mecanismos que permiten aplicar la teoría.

En general, este documento aborda un proceso de valoración establecido a partir de la Norma Internacional de Contabilidad 36 empleando la metodología borrosa. Por lo tanto, se hace necesario determinar si el proceso utilizado es aplicable al objetivo que se pretende, si los datos básicos pueden ser obtenidos y si el proceso empleado tiene un rango de fiabilidad esperado con relación a los datos obtenidos (Mattessich, 2002).

Para abordar las anteriores consideraciones, se hace fundamental detallar los conceptos y procedimientos empleados en el proceso experimental, que viabilizan y justifican la correlación entre los conceptos. A continuación, se presentan los conceptos de la teoría de los subconjuntos borrosos sobre los cuales se realiza el tratamiento de la variable y se describe el proceso que permite correlacionar la variable y la teoría presentada.

\subsection{La teoría de los subconjuntos borrosos $^{2}$}

La teoría de los subconjuntos borrosos, planteada por Lotfi Aliasker Zadeh (1965), surge como una herramienta adecuada para la representación de situaciones aproximadas por naturaleza (Franco de los Ríos, 2012). En particular, permite considerar y tratar la incertidumbre epistémica descrita.

A continuación se describen algunos de los conceptos más importantes de la teoría y empleados en los propósitos del presente documento.

\section{Definición 1}

Sea un universo $X$ continuo o discreto, un subconjunto borroso Ã es una función $\mu_{\tilde{\mathrm{A}}}: X \rightarrow[0,1]$ que asigna a cada elemento del conjunto $X$ un valor $\mu_{\tilde{\AA}}(x)$ perteneciente al intervalo $[0,1]$, llamado grado o nivel de pertenencia de $x$ a $\tilde{A}$ (Lazzari, 2010, p. 98).

Donde Ã denota tanto al subconjunto como a la función de pertenencia asociada, es decir, un subconjunto borroso Ã está perfectamente caracterizado por los pares ordenados $\tilde{\mathrm{A}}=\left\{\left(\mathrm{x}, \mu_{\tilde{\mathrm{A}}}\right), x \in X\right\}$. Los conjuntos nítidos pueden expresarse como un caso particular de los subconjuntos borrosos. Por ejemplo: la función de pertenencia de un intervalo cerrado $[a, b]$ de números reales (Lazzari, 2010, p. 99) es:

$$
\forall x \in \mathbb{R}: \mu_{\tilde{A}}(x)\left\{\begin{array}{c}
1 \text { si } x \in[a, b] \\
0 \text { en otro caso }
\end{array}\right.
$$

\footnotetext{
2 Para una ampliación sobre la teoría de los subconjuntos borrosos, sus propiedades, aritmética y formalización, se pueden consultar: James J. Buckley, Esfandiar Eslami y Thomas Feuring (2002), Fabián Alberto Castiblanco-Ruiz (2016), Arnold Kaufmann y Jaime Gil-Aluja (1987, 1993), George J. Klir y Bo Yuan (1995), Luisa Lucila Lazzari (2010).
} 
Es decir, para establecer un subconjunto borroso se fijan los elementos y sus correspondientes valores o grado de pertenencia.

\section{Definición 2}

Intervalo de confianza. Dados dos números reales $a_{1}$ y $a_{2}$, tales que $a_{1} \leq a_{2}$, se llama intervalo cerrado de extremos $a_{1}$ y $a_{2}$ al conjunto de números reales $(\mathbb{R})$.

$\left[a_{1}, a_{2}\right]=\left\{x: x \in \mathbb{R} \wedge a_{1} \leq x \leq a_{2}\right\}$

$a_{1}$ es el extremo inferior o izquierdo y $a_{2}$ es el extremo superior o derecho (Lazzari, 2010, p. 114).

A partir de los conceptos de subconjunto borroso e intervalo de confianza, los números borrosos se pueden definir de dos maneras equivalentes; estas definiciones permiten representar un mismo número borroso. Se define según lo expuesto por Arnold Kaufmann y Jaime Gil-Aluja (1987, p. 43).

\section{Definición 3}

a. Un número borroso Ã es un subconjunto borroso normal y convexo de $\mathbb{R}$, es decir, aquel cuya función de pertenencia $\mu_{\tilde{\AA}}(x)$ es normal y convexa.

b. Un número borroso Ã se halla formado por una secuencia finita o infinita de intervalos de confianza con las siguientes propiedades:

1. Se afecta a cada intervalo de confianza un valor $\alpha \in[0,1]$, de tal manera que dos intervalos de confianza diferentes no pueden tener el mismo valor $\alpha$. Dicho valor $\alpha$ se denomina "nivel de presunción".
2. Si se designa por $\mathrm{A}_{\alpha}=\left[a_{1}(\alpha), a_{2}(\alpha)\right]$ el intervalo de confianza de nivel $\alpha$. Se debe cumplir $\left(\alpha^{\prime}, \alpha\right) \Rightarrow\left(A_{\alpha} \supset A_{\alpha^{\prime}}\right)$. Dicho de otra manera, los intervalos de confianza deben encajarse, estrictamente o no, los unos con los otros.

3. Hay un intervalo y solo uno que puede reducirse a un real único.

Por lo tanto, un número borroso Ã es una generalización del concepto de intervalo de confianza; es decir, se considera una familia de intervalos que cumplen las condiciones 1 ), 2 ) y 3); el intervalo de confianza de nivel $\alpha$ se designa por $A_{\alpha}$ y se denomina " $\alpha$-corte de $A$ ".

En conclusión, un número borroso Ã puede ser representado de dos maneras:

1. Por medio de $\alpha$-cortes, $\forall \alpha \in[0,1]$ denotado $A_{\alpha}$.

2. Por su función de pertenencia $\mu_{\tilde{\AA}}(x)$.

En este sentido, los números borrosos al ser subconjuntos borrosos, se pueden representar por sus $\alpha$-cortes de manera única; en particular, al ser subconjuntos borrosos convexos, todos los $\alpha$-cortes son intervalos cerrados de números reales (Lazzari, 2010, p. 118).

Los números borrosos triangulares (NBT) son una clase particular de números borrosos, caracterizados por poseer función de pertenencia de tipo lineal, y cuya representación gráfica forma un triángulo con el eje $x$. Por lo tanto, todo NBT Ã posee una función $\mu_{\tilde{A}}(x)$ de la siguiente forma:

Para todo $x \in \mathbb{R}$, se define la función, 


$$
\mu_{\tilde{A}}(x)=\left\{\begin{array}{c}
0 \text { si } x \leq a_{1} \\
\frac{x+a_{1}}{a_{2}-a_{1}} \text { si } a_{1} \leq x \leq a_{2} \\
\frac{-x+a_{3}}{a_{3}-a_{1}} \text { si } a_{2} \leq x \leq a_{3} \\
0 \text { si } a_{3} \leq x
\end{array}\right.
$$

O mediante sus $\alpha$-cortes:

$$
\mathrm{A}_{\alpha}=\left[\left(a_{2}-a_{1}\right) \alpha+a_{1},-\left(a_{3}-a_{2}\right) \alpha+a_{3}\right]
$$

Es decir, un NBT queda perfectamente identificado por los tres valores $a_{1}, a_{2}, a_{3}$, y por lo tanto, puede ser representado como:

$\tilde{\mathrm{A}}=\left[a_{1}, a_{2}, a_{3}\right]$ donde $a_{1} \leq a_{2} \leq a_{3}$

Por lo tanto, para el NBT definido por $A_{a}=[3 \alpha+2,-3 \alpha+8]$ o con función de pertenencia,

$$
\Omega(x)=\left\{\begin{array}{c}
0 \text { si } x \leq 2 \\
\frac{x+2}{3} \text { si } 2 \leq x \leq 5 \\
\frac{-x+8}{3} \text { si } 5 \leq x \leq 8 \\
0 \text { si } 8 \leq x
\end{array}\right.
$$

Se tiene la representación dada por $\tilde{\mathrm{A}}=[2,5,8]$.

En esta representación $a_{1}=2$ y $a_{3}=8$ designan el valor mínimo y el valor máximo, es decir, con nivel de pertenencia $\alpha=0$, mientras que $a_{2}=5$ designa el valor con mayor nivel de pertenencia, es decir, $\alpha=1$. Su representación gráfica aparece en el gráfico 1.

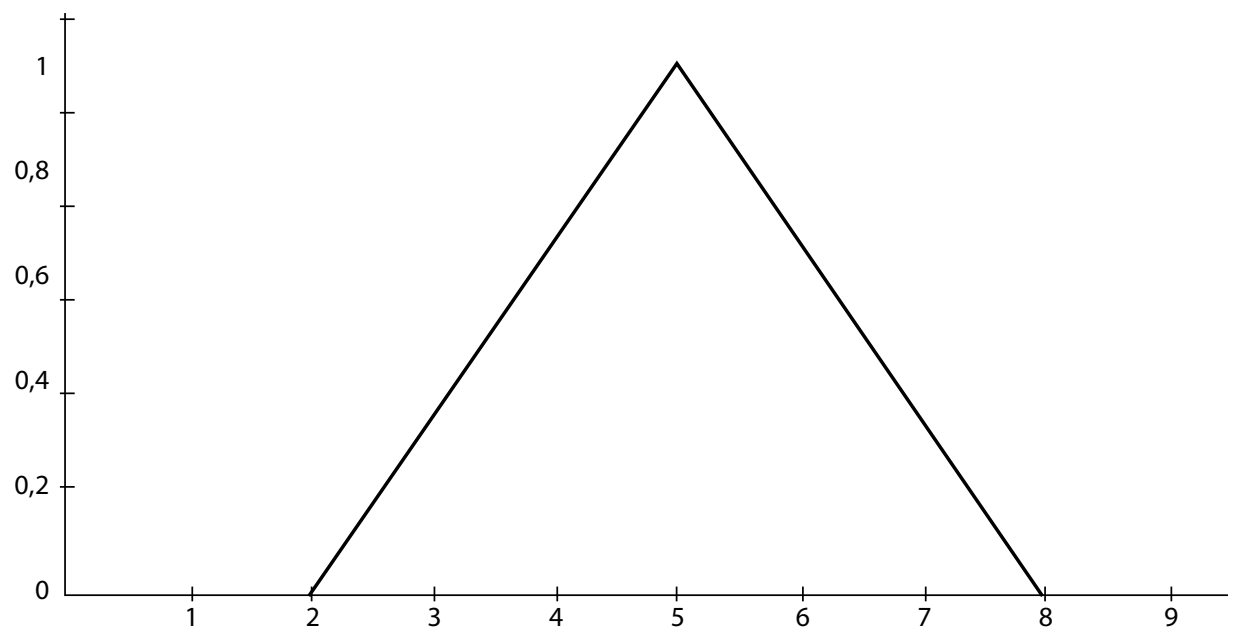

Gráfico 1

Número triangular borroso $[2,5,8]$

Fuente: elaboración propia 
A partir de esta representación para los NBT, las operaciones básicas se obtienen como muestra el siguiente ejemplo:

Dados $\tilde{A}$ y $\tilde{B}$ dos NBT representados mediante $\tilde{\mathrm{A}}=[2,3,8]$ y $\tilde{B}=[5,7,9]$

$\tilde{\mathrm{A}}=\tilde{B}=[2,3,8]+[5,7,9]=[2+5,3+7,8+$ 9] $=[7,10,17]$

$\tilde{\mathrm{A}}-\tilde{B}=[2,3,8]+[5,7,9]=[2+9,3-7,8-5]$ $=[7,4,3]$

Para $k=3,3 \tilde{\mathrm{A}}=[\operatorname{Min}(6,24), 3(3), \operatorname{Max}[6$, $24)]=6,924]$

Sin embargo, la multiplicación y la división entre dos NBT no siempre dan como resultado un NBT, es decir, con funciones lineales; por lo tanto, estas operaciones entre los mismos deberán ser desarrolladas a partir de sus representaciones como $\alpha$ - cortes, para obtener el número borroso no triangular. El número borroso obtenido contendrá igualmente dos valores extremos, límites de las posibilidades y un valor central que designa el dato con mayor nivel de posibilidad; sin embargo, sus funciones no serán lineales, sino funciones de segundo grado (en el caso de la multiplicación), manteniendo las propiedades de convexidad y normalidad, características de un numero borroso.

\subsection{Proceso de borrosificación y desborrosificación}

Se analiza la variable flujo de caja proyectado bajos las categorías establecidas (incertidumbre y subjetividad), para determinar si bajo ciertas condiciones se cumplen las características de incertidumbre epistémica descrita. Ante tal situación, se emplea el proceso de borrosificación, el cual permite establecer un número bo- rroso a partir de la aproximación o descripción lingüística dada para la variable en cuestión. Es decir, se realiza un proceso de conversión de la variable a partir de datos inexactos, estimaciones subjetivas o cargadas de incertidumbre epistémica, en un dato borroso. Por lo general, este proceso se realiza mediante la conversión de los valores reales dados para la variable, como aproximaciones borrosas reales de este número. Tal método de borrosificación se conoce con el nombre de singleton (Martín del Brío \& Sanz-Molina, 2002).

Por ejemplo, si se estima que el valor del flujo de caja proyectado para cierto período se va a encontrar entre 120 unidades monetarias (u.m.) y 175 u.m., y 156 u.m. es el valor con mayor posibilidad, estos tres valores permiten determinar en particular un NBT que sería expresado como [120, 156, 175]. Nótese que bajo la información planteada no es conveniente ni posible establecer con certeza una distribución de probabilidad. En la sección de resultados se describirá el procedimiento empleado.

Borrosificada la información, se aplican las operaciones definidas para los NBT en el cálculo del valor presente del flujo de caja proyectado; el resultado será nuevamente un dato borroso mediante el cual se expresa la inexactitud e incertidumbre de la variable trabajada.

Sin embargo, en muchas ocasiones se hace necesaria la estimación de la variable incierta mediante un valor cierto, es decir, mediante la asignación de un valor real. Este proceso es conocido como desborrosificación de números borrosos. Hay diferentes métodos para la obtención de números reales a partir de números borrosos; Jorge de Andrés-Sánchez (2000) destaca los mé- 
todos clásicos (media borrosa y centro de área), el indicador de valor de Miguel Delgado CalvoFlores (Delgado Calvo-Flores, Vila \& Woxman, 1998) y el valor esperado de un número borroso.

La desborrosificación de un NBT mediante el cálculo del valor esperado es uno de los más empleados debido a su gran simplicidad. Dado un NBT $\tilde{\mathrm{A}}=\left[a_{1}, a_{2}, a_{3}\right]$, donde $a_{1} \leq a_{2} \leq a_{3}$, el valor esperado de $\tilde{\mathrm{A}}$ se define como:

$V E[\tilde{A}]=\frac{a_{1}+2 a_{2}+a_{3}}{4}$

Para el ejemplo dado anteriormente, se tendrá que el valor esperado es:

$$
V E[\tilde{A}]=\frac{120+2(156)+175}{4}=151,75
$$

Este valor difiere claramente del promedio o media aritmética de los tres valores que definen el NBT. Este hecho particular se debe a la asimetría del NBT.

El proceso de desborrosificación se hace pertinente y necesario bajo dos perspectivas. Por un lado, es oportuno establecer un valor que permita comparar los datos obtenidos por medio de la metodología borrosa con otras metodologías, lo cual solo es posible mediante cantidades de la misma naturaleza (números reales). Por otro lado, se hace necesario mientras las directivas y algunos aspectos normativos así lo exijan.

\section{Resultados}

El cálculo del valor presente de cantidades estimadas, según las condiciones de incertidum- bre inherentes, puede ser establecido a partir de conjeturas, basadas en las expectativas de la situación particular o de técnicas estadísticas. Sin embargo, cuando la información disponible se halla en el ámbito de la incertidumbre epistémica, se hace viable un proceso de borrosificación que permita contemplar y tratar esta incertidumbre. En James J. Buckley, Esfandiar Eslami y Thomas Feuring (2002) y en Arnold Kaufmann y Jaime Gil-Aluja (1987) se establecen los procedimientos para el cálculo del valor presente de cantidades estimadas desde una perspectiva borrosa, incluyendo flujos de dinero borroso y tasas de interés borrosas.

En particular, en el presente trabajo se busca aplicar la metodología borrosa en la determinación del valor de uso de un activo o UGE, lo cual se ejemplifica mediante la siguiente situación:

Se ha determinado que los flujos de efectivo generados por un activo o UGE para los próximos cuatro (4) años de cierta organización están dados por las siguientes expresiones: para el primer año, el importe estimado está en algún valor entre 70 u.m. y 200 u.m., y 150 u.m. es el más posible. Para el segundo año, el importe estimado está entre 80 u.m. y 210 u.m., y 170 u.m. es el más posible. Para el tercer año, el valor estará entre 85 u.m. y 220 u.m., y 180 u.m. es el valor con mayor posibilidad de acaecimiento. Finalmente, para el cuarto año, el valor estimado estará entre 90 u.m. y 225 u.m., y 195 u.m. es el más posible. Se solicita hallar el valor de uso del activo o UGE correspondiente, con una tasa de descuento del 7\%. Se consideran para el activo un valor razonable de 530 u.m. y un valor en libros de 560 u.m. 
Identificada la incertidumbre epistémica, dada por el contexto (información limitada para establecer una función de probabilidad e información inexacta y aproximada) se lleva a cabo el proceso de borrosificación. Para tal fin, expresiones como: para el primer año, el importe estimado está en algún valor entre 70 u.m. y 200 u.m., y 150 u.m. es el más posible plantean que la estimación sobre el flujo de caja está con un alto grado de proximidad al valor de 150 u.m., es decir, el valor estimado aunque se pueda hallar entre 70 u.m. y 200 u.m, se encuentra más cercano al valor de 150 u.m. Por lo tanto, si el valor de 1 representa absoluta cercanía a 150 y 0 representa no proximidad a 150 , se puede establecer una función a trozos con rango entre 0 y 1 que represente, para los demás valores de manera gradual, la proximidad al valor con mayor posibilidad.

De forma clara, la cercanía al valor de 150 u.m. se puede establecer mediante una relación lineal entre los valores estimados y el intervalo [0,1]; a mayor cercanía a 150 u.m., mayor cercanía a 1. Por lo tanto, la función que modela tal situación es:

$$
\mu_{p}=\left\{\begin{array}{c}
0 \text { si } x \leq 70 \\
\frac{x-70}{80} \text { si } 70 \leq x \leq 150 \\
\frac{-x+200}{50} \text { si } 150 \leq x \leq 200 \\
0 \text { si } x \geq 200
\end{array}\right.
$$

Así pues, a manera de ejemplo, una estimación para el flujo de efectivo esperado de 160 u.m. tiene un grado de pertenencia, dado por: $\mu_{\tilde{p}}(160)=\frac{-160+200}{50}=0,8$

Mientras que una estimación de 90 u.m. tiene un grado de pertenencia dado por:

$\mu_{\tilde{p}}(90)=\frac{90-70}{80}=0,25$

De manera clara, conforme un valor contemplado en la estimación se aleja del valor con mayor posibilidad, disminuye su grado de pertenencia. Sin embargo, sigue siendo contemplado para el análisis.

Con lo anterior, a la estimación planteada se le ha asignado el NBT $\tilde{P}=[70,150,200]$ mediante sus valores reales, es decir, mediante el singleton. Las demás estimaciones borrosificadas se presentan en la tabla 1.

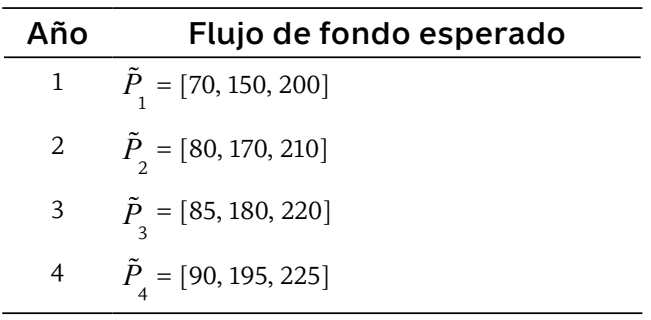

Tabla 1

Flujos de fondo esperados como NBT

Fuente: elaboración propia

El proceso de borrosificación pudo haberse establecido incluso a partir de la información plasmada en los presupuestos financieros desde una perspectiva borrosa. Fuencisla MartínezLobato y Máximo Ferrando-Bolado (1997) y Fabián Alberto Castiblanco-Ruiz (2014, 2016) plantean la aplicación de la teoría de los subconjuntos borrosos en el establecimiento de los presupuestos de las organizaciones. Con esto, la borrosificación de los datos inciertos se hereda 
y se asume desde el proceso de planificación financiera.

Ahora bien, considerados los flujos de caja esperados como NBT se procede al cálculo del valor presente, según las operaciones definidas sobre NBT.

$$
\begin{aligned}
& \widetilde{V P}_{1}=\frac{1}{(1+0.07)^{1}}([70,150,200])=[65.42,140.18,186.9] \\
& \widetilde{V P}_{2}=\frac{1}{(1+0.07)^{2}}([80,170,210])=[69.87,148.48,183.42] \\
& \widetilde{V P}_{3}=\frac{1}{(1+0.07)^{3}}([85,180,220])=[69.38,146.93,179.58] \\
& \widetilde{V P}_{4}=\frac{1}{(1+0.07)^{4}}([90,195,225])=[68.66,148.76,171.65]
\end{aligned}
$$

\section{Con esto se obtiene:}

Valor presente de flujo de efectivo esperado borroso: $\widetilde{V P}=[273.33,584.35,721.55]$, el cual es equivalente al valor de uso del activo o UGE.

Obtenido el NBT que contempla la incertidumbre y la subjetividad propia de la estimación planteada, se hace necesario aplicar el proceso de desborrosificación de tal forma que se asocie a un número real representativo del NBT. Para este proceso, se emplea el método del valor esperado para un NBT. Por lo tanto, el valor después de este proceso será (denotado como $\left.V_{d}\right)$ :

Valor de uso del activo o UGE: $V_{d}=1 / 4(273.33+2(273.33+2(584.35)+$ 721.55) $=540.9$

En primera instancia, debido a la construcción de la teoría de los subconjuntos borrosos y su sustento matemático, la incertidumbre y la subjetividad quedan contempladas dentro del tratamiento de la información y se vislumbran todos los posibles escenarios de acaecimien- to de los sucesos. Por otro lado, se puede inferir de lo planteado en el Apéndice A de la NIC 36, que la idea es establecer "una mejor estimación del valor en uso que los importes mínimo, más probable o máximo tomados de forma aislada" (párrafo A11). Por lo tanto, la metodología aquí expuesta cumple con dicho propósito. Si bien el promedio como herramienta propuesta por la norma, establece una medida de tendencia central que engloba los datos trabajados, por su naturaleza no contempla la incertidumbre inherente del suceso observado.

Por su parte, los números borrosos permiten considerar y tratar la incertidumbre sin reducirla precipitadamente a un valor. Quizás después de contemplar la incertidumbre, modelarla y operar con ella, sea oportuno establecer un proceso de agregación o desborrosificación de la información; sin embargo, lo erróneo sería trabajar con un dato agregado (como la media aritmética) desde el inicio de cualquier proceso en el marco de la incertidumbre epistémica.

Es decir, si se compara el valor de uso hallado mediante la metodología borrosa con el valor de uso empleando las estimaciones dadas por la norma, se encuentra la siguiente diferencia; la norma propone hallar la relación entre el máximo, el mínimo y el valor más posible mediante el promedio de los tres valores, es decir, para el ejemplo expuesto se tiene: el flujo de efectivo esperado para el primer período es $F_{1}=\left(\frac{70+150+200}{3}\right)=140$ con lo cual su valor presente correspondiente sería $V P_{1}=\frac{140}{(1+0.07)^{1}}=130.84$. Repitiendo este proceso para los flujos de efectivo de los siguientes períodos, se obtiene (se denota $V_{m}$ ): 
Valor de uso del activo o UGE: $V_{m}=526.33$ u.m.

Bajo esta metodología se obtiene un menor valor de uso y por consiguiente, según los datos planteados en la situación específica, no se tomaría para determinar el valor de deterioro. En particular, el deterioro del valor del activo o UGE sería mayor. La tabla 2 muestra la comparación entre las dos metodologías empleadas.

\begin{tabular}{|c|c|c|}
\hline Información & $\begin{array}{c}\text { Metodología } \\
\text { por } \\
\text { promedio }\end{array}$ & $\begin{array}{c}\text { Metodología } \\
\text { borrosa }\end{array}$ \\
\hline Valor en libros & 560 u.m. & 560 u.m. \\
\hline Valor razonable & 530 u.m. & 530 u.m. \\
\hline Valor de uso & 526.33 u.m. & 540.9 u.m. \\
\hline Valor recuperable & 530 u.m. & 540.9 u.m. \\
\hline $\begin{array}{l}\text { Deterioro del valor } \\
\text { del activo o UGE }\end{array}$ & 30 u.m. & 19.1 u.m. \\
\hline
\end{tabular}

Tabla 2

Comparación entre metodologías empleadas para determinar el deterioro del valor un activo o UGE

Fuente: elaboración propia

Deterioro del activo (metodología por promedio $)=560-530=30$

Deterioro del activo (metodología borrosa) $=560-540.9=19.1$

La diferencia sustancial en los valores encontrados radica en el siguiente hecho: cuando se establece la estimación a partir del enunciado, para el primer año el importe estimado está en algún valor entre 70 u.m. y 200 u.m., y 150 u.m. es el más posible, se hace evidente que las diferencias por encima y por debajo del valor más posible no son iguales, con lo cual al tomar el promedio entre las cifras, esa diferencia se deprecia.
Bajo la metodología borrosa, se considera que estos diferenciales representados mediante la asimetría del NBT, son parte constitutiva y esencial de la imperfección e inexactitud de la información tratada. Por lo tanto, la estimación considerada como un NBT contempla la incertidumbre epistémica inherente al tipo de datos $y$, por ende, permite un tratamiento de la información de manera más fiable sin depreciar la asimetría de la información dada.

\section{Conclusiones}

El proceso de estimación de valores localizados en el futuro conlleva - de manera inevitableabordar y tratar la incertidumbre propia de los fenómenos económicos y financieros en prospectiva. Esta situación pone de manifiesto la existencia de diversas tipologías sobre la incertidumbre; en primer lugar, se plantea un tipo de incertidumbre aleatoria, bajo la cual son conocidos los posibles eventos que resultan de un acontecimiento, es decir, se puede establecer una función de probabilidad que modele el escenario futuro.

Por otra parte, se plantea una incertidumbre epistémica, que hace referencia a la ausencia de certeza o conocimiento seguro. En esta categoría, referida en particular al conocimiento, están conceptos como la ambigüedad, la vaguedad y la imprecisión. En particular, se refiere a la dificultad e, incluso, a la imposibilidad de establecer distribuciones de probabilidad a los acontecimientos.

Ante la incertidumbre epistémica, los números borrosos se adaptan en particular al establecimiento de pronósticos y presupuestos en 
la organización, pues describen de manera amplia y concreta las estimaciones planteadas para tal proceso, sin dejar de lado la incertidumbre latente en el estudio de hechos o variables localizadas en el futuro.

El concepto de valor de uso de un activo o unidad generadora de efectivo, que permite en variadas ocasiones establecer el deterioro de su valor, pone en juego magnitudes confinadas en el futuro y, por ende, cargadas de incertidumbre. En particular, los flujos de efectivo esperado son variables inmersas en un contexto incierto o subjetivo. Ante tal situación, la NIC 36 establece algunas situaciones y las posibles técnicas que permiten abordar tales contextos. Sin embargo, la manera como se aborda la incertidumbre epistémica no permite siempre establecer estimaciones que reflejen de manera representativa la información tratada.

Frente a la anterior situación, la metodología borrosa se convierte en una herramienta alternativa que permite considerar la imperfección e inexactitud de las estimaciones dadas. Esta metodología establece valores más cercanos en cuanto a la evolución en términos financieros de los activos o UGE, por cuanto no excluye ni distribuye siempre en igual proporción los posibles datos de un evento estimado.

Con lo anterior, se evita en gran medida devaluar o sobrevalorar los activos o UGE, por limitaciones en la información recopilada, es decir, por desechar la incertidumbre.

Si bien la metodología propuesta no permite reducir la incertidumbre ni la subjetividad propia de la información disponible, sí permite su consideración y tratamiento. Bajo la propuesta abordada no es posible pensar en una mitigación, disminución ni mucho menos despreciar la incertidumbre propia de los sucesos; por eso, se plantea una metodología que propenda por su consideración y abordaje en diversos procesos. En otras palabras, se busca operar con incertidumbre.

Conforme aumenta el tiempo de estimación a futuro y mientras se opere con valores cargados de incertidumbre y subjetividad, estas mismas categorías van aumentando; por lo tanto, se hace fundamental una herramienta que permita operar con valores inciertos y ver la amplitud de posibilidades en el comportamiento de variables cargadas de incertidumbre y subjetividad.

\section{Referencias}

Andrés-Sánchez, Jorge de (2000). Estimación de la estructura temporal de los tipos de interés mediante números borrosos. Aplicación a la valoración financiero-actuarial y el análisis de la solvencia del asegurador de vida. Tesis de doctorado no publicada. Universidad Rovira i Virgili, Tarragona, España.

Disponible en: http://www.tesisenred.net/ handle/10803/8804

Buckley, James J.; Eslami, Esfandiar \& Feuring, Thomas (2002). Fuzzy Mathematics in Economics and Engineering. New York: Springer-Verlag Berlin Heidelberg.

Cárdenas y Nápoles, Raúl Andrés (2002). Presupuestos. Teoría y práctica. Ciudad de México: McGraw-Hill.

Castiblanco-Ruiz, Fabián Alberto (2014). Una mirada al presupuesto anual de ventas de Rautenstrauch y Villers a partir de 
los números borrosos: el manejo de la incertidumbre y la subjetividad. Criterio Libre, 12 (20), 199-222. Disponible en: http://www.unilibre.edu.co/CriterioLibre/ images/revistas/20/12-Revista-Criterio\%20 Libre\%20V12-20-Articulo\%209.pdf Castiblanco-Ruiz, Fabián Alberto (2016). La teoría de los subconjuntos borrosos en el proceso presupuestario de las organizaciones. Bogotá: Editorial Universitaria de la Universidad La Gran Colombia.

Delgado Calvo-Flores, Miguel; Vila, M. Amparo \& Woxman, William (1998). On a Canonical Representation of Fuzzy Numbers. Fuzzy Sets and Systems, 93, 125135.

Franco de los Ríos, Camilo A. (2012). Modelización de relaciones de preferencia: una aproximación borrosa y bipolar al proceso subjetivo de decisión bajo incertidumbre. Tesis Doctoral no publicada. Universidad Complutense de Madrid, Madrid, España. Disponible en: http://eprints.ucm. es/16448/1/T33900.pdf

Gil-Aluja, Jaime (2000). Génesis de una teoría de la incertidumbre. Encuentros Multidisciplinares, 2 (6), 1-8. Disponible en: http://www.encuentros-multidisciplinares. org/Revistan\%BA6/Jaime\%20Gil\%20 Aluja\%201.pdf

International Financial Reporting Standards Foundation, IFRS Foundation (2014). Norma Internacional de Contabilidad No. 36: Deterioro del Valor de los Activos. En Normas Internacionales de Información Financiera, IASB.
Kaufmann, Arnold \& Gil-Aluja, Jaime (1987). Técnicas operativas de gestión para el tratamiento de la incertidumbre. Barcelona: Hispano Europea, S.A.

Kaufmann, Arnold \& Gil-Aluja, Jaime (1993). Introducción de la teoría de los subconjuntos borrosos a la gestión de la empresa. Santiago de Compostela: Milladoiro.

Klir, George J. \& Yuan, Bo (1995). Fuzzy Sets and Fuzzy Logic. New Jersey: Prentice Hall. Lazzari, Luisa Lucila (2010). El comportamiento del consumidor desde una perspectiva fuzzy. Una aplicación al turismo. Buenos Aires: EDICON, Fondo Editorial Consejo.

Martín del Brío, Bonifacio \& Sanz-Molina, Alfredo (2002). Redes neuronales y sistemas difusos. Ciudad de México: Alfaomega.

Martínez-Lobato, Fuencisla \& FerrandoBolado, Máximo (octubre-diciembre de 1997). Un modelo de simulación borroso de planificación financiera. Revista Española de Financiación y Contabilidad, XXVI (93), 1091-1123.

Mattessich, Richard (2002). Contabilidady métodos analíticos. Medición y proyección de la riqueza en la microeconomía y en la macroeconomía. Buenos Aires: La Ley.

Pérez, Rodolfo H. (1999). Epistemología de la incertidumbre. Madrid: Real Academia de Ciencias Económicas y Financieras, RACEF. Disponible en: https://racef.es/archivos/ discursos/160_perez1999.pdf

Ramírez-Sarrió, Dídac (1988). Fundamentos metodológicos para el análisis económico en contextos de incertidumbre. Barcelona: Universitat de Barcelona. Disponible en: http://www.tdx.cat/handle/10803/96662 
Torres, Álvaro \& Tranchita, Carolina (2004). ¿Inferencia y razonamiento probabilístico o difuso? Revista de Ingeniería, 19, 157165. Disponible en: https://ojsrevistaing. uniandes.edu.co/ojs/index.php/revista/ article/view/450/652

Zadeh, Lotfi Aliasker (1965). Fuzzy Sets. Information and Control, 8, 333-353. Disponible en: https://people.eecs.berkeley. edu/ zadeh/papers/Fuzzy\%20SetsInformation\%20and\%20Control-1965.pdf
- Fecha de recepción: 22 de abril de 2016

- Fecha de aceptación: 29 de julio de 2016

- Disponible en línea: 14 de diciembre de 2016

\section{Para citar este artículo}

Castiblanco-Ruiz, Fabián Alberto (2016). Valor de uso de un activo o unidad generadora de efectivo bajo incertidumbre: el flujo de efectivo esperado mediante metodología borrosa. Cuadernos de Contabilidad, 17 (44), 449-465. https://doi. org/10.11144/Javeriana.cc17-44.vuau 
